\title{
Knockdown of long non-coding RNA HOTAIR reverses cisplatin resistance of ovarian cancer cells through inhibiting miR-138-5p-regulated EZH2 and SIRT1
}

\author{
Yun Zhang ${ }^{1,2}$, Hao $\mathrm{Ai}^{1,2}$, Xue Fan ${ }^{3}$, Suxian Chen ${ }^{4}$, Yadi Wang ${ }^{5}$ and Lili Liu ${ }^{1 *}$
}

\begin{abstract}
Background: Cisplatin resistance (DDP-resistance) remains one of the major causes of poor prognosis in females with ovarian cancer. Long non-coding RNAs (IncRNAs) have been shown to participate in the regulation of cellular processes, including chemoresistance. The aim of this study was to explore the role of HOX transcript antisense RNA (HOTAIR) in DDP-resistant ovarian cancer cells.

Methods: DDP-resistant ovarian cancer cell lines (SKOV3/DDP and A2780/DDP) were established. Real-time PCR, western blot, dual-luciferase reporter assay, and flow cytometry were then used to evaluate the effect of HOTAIR/miR138-5p axis on chemoresistance of DDP-resistant ovarian cancer cells to DDP.

Results: We found that HOTAIR was upregulated in DDP-resistant cells, while miR-138-5p was downregulated. Knockdown of HOTAIR increased the expression of miR-138-5p in DDP-resistant cells and miR-138-5p is directly bound to HOTAIR. Upregulation of miR-138-5p induced by HOTAIR siRNA or by its mimics enhanced the chemosensitivity of DDP-resistant cells and decreased the expression of EZH2 (enhancer of zeste 2 polycomb repressive complex 2 subunit) and SIRT1 (sirtuin 1). Furthermore, the HOTAIR silencing-induced chemosensitivity of DDP-resistant cells was weakened by miR-138-5p inhibitor.
\end{abstract}

Conclusions: These data demonstrate that HOTAIR acts as a sponge of miR-138-5p to prevent its binding to EZH2 and SIRT1, thereby promoting DDP-resistance of ovarian cancer cells. Our work will shed light on the development of therapeutic strategies for ovarian cancer treatment.

Keywords: Ovarian cancer, DDP resistance, HOTAIR, miR-138-5p

\section{Background}

Ovarian cancer, one of the most lethal diseases in the female reproductive system, is responsible for $4 \%$ of deaths from cancer in women [1]. Ovarian cancer can be divided into three broad subgroups: epithelial, stromal,

\footnotetext{
*Correspondence: clearsky0315@sina.com

${ }^{1}$ Department of Obstetrics and Gynecology, The First Affiliated

Hospital of Jinzhou Medical University, No. 2, Section 5, Renmin Street, Jinzhou 121001, Liaoning, People's Republic of China

Full list of author information is available at the end of the article
}

and germ cell tumors, of which epithelial ovarian cancer is the most lethal type of ovarian cancer and accounts for $85 \%$ of all reported cases [2]. Cisplatin (DDP) is one of the first line agents employed in the treatment of epithelial ovarian cancer [3]. However, DDP-resistance is frequently observed in advanced epithelial ovarian cancer patients and predicts poor prognosis [4]. Therefore, it is important to investigate the molecular basis of DDPresistance in ovarian cancer and identify more effective therapeutic strategies.

(c) The Author(s) 2020. This article is licensed under a Creative Commons Attribution 4.0 International License, which permits use, sharing, adaptation, distribution and reproduction in any medium or format, as long as you give appropriate credit to the original author(s) and the source, provide a link to the Creative Commons licence, and indicate if changes were made. The images or other third party material in this article are included in the article's Creative Commons licence, unless indicated otherwise in a credit line to the material. If material is not included in the article's Creative Commons licence and your intended use is not permitted by statutory regulation or exceeds the permitted use, you will need to obtain permission directly from the copyright holder. To view a copy of this licence, visit http://creativeco mmons.org/licenses/by/4.0/. The Creative Commons Public Domain Dedication waiver (http://creativecommons.org/publicdomain/ zero/1.0/) applies to the data made available in this article, unless otherwise stated in a credit line to the data. 
Long non-coding RNAs (lncRNAs), a class of non-coding transcripts, have recently been reported as important regulators of cell proliferation, invasion, and apoptosis in several cancer types [5-7]. Moreover, multiple lines of evidences showed that lncRNAs were dysregulated in various types of cancers [8-10]. The HOX transcript antisense RNA (HOTAIR) gene has been identified and located within the Homeobox $\mathrm{C}$ (HOXC) gene cluster on Chromosome 12 and encodes a $2.2 \mathrm{~kb}$ lncRNA molecule [11]. HOTAIR expression was initially found to be upregulated in primary breast tumors and metastases [12]. In recent studies, upregulation of HOTAIR has been proven to be associated with the metastasis of various malignant tumors, such as colorectal cancer [13], hepatocellular carcinoma [8], and pancreatic carcinoma [14]. Moreover, a relatively small number of studies have associated HOTAIR with ovarian cancer. Although recent studies found that overexpression of HOTAIR could lead to chemoresistance in ovarian cancer $[15,16]$, the underlying molecular mechanism needs to be further investigated.

MicroRNA-138-5p (miR-138-5p), a non-coding small RNA molecule which only expressed in the ovaries, was recently identified as a cancer suppressor by post-transcriptionally repressing the expression of proto-oncogenes [17-19]. Unfortunately, although the potential effectiveness was identified in hepatocellular carcinoma [20], non-small cell lung cancer [21] and nasopharyngeal carcinoma [22], the role of miR-138-5p involved in DDP resistance of ovarian cancer cells needs to be addressed. Moreover, there is no report about the correlation between HOTAIR and miR-138-5p on regulating DDP resistance in ovarian cancer cells.

In this study, we detected the expression of HOTAIR and miR-138-5p in DDP-resistant cells and investigated correlation effects of HOTAIR and miR-138-5p in DDP resistant ovarian cancer cells.

\section{Materials and methods}

\section{Cell culture and transfection}

Two ovarian cancer cell lines, SKOV3 and A2780 were purchased from Procell Life Science \&Technology Co., Ltd. (Wuhan, China), cultured in Dulbecco's modified Eagle's medium (Sigma, St. Louis, MO, USA) containing $10 \%$ fetal bovine serum (Sigma), and maintained at $37{ }^{\circ} \mathrm{C}$ with $5 \% \mathrm{CO}_{2}$. Drug-resistant cell lines of SKOV3 and A2780 were constructed by treatment of proliferating cell cultures with DDP (Dalian Meilun Biotechnology Co., Ltd., Dalian, China) at final concentrations of $8 \mu \mathrm{M}$ for 12 weeks.

Drug-resistant cells were seeded into 6-well plates and transfected with miR-138-5p mimic, negative control (NC) mimic (a non-specific miRNA mimic), miR-138-5p
Table 1 The sequences of interference vectors

\begin{tabular}{ll}
\hline Name & Sequence \\
\hline si-HOTAIR-1 & 5'-UAACAAGACCAGAGAGCUGTT-3' \\
5i-HOTAIR-2 & 5'-CAGCUCUCUGGUCUUGUUATT-3' \\
& 5'-GAACGGGAGUACAGAGAGATT-3' \\
NC siRNA & 5'-UUCUCCGAACGUGUCACGUTT-3' \\
& 5'-ACGUGACACGUUCGGAGAATT-3' \\
mir-138-5p mimics & 5'-AGCUGGUGUUGUGAAUCAGGCCG-3' \\
& 5'-GCCUGAUUCACAACACCAGCUUU-3' \\
NC mimics & 5'-UUCUCCGAACGUGUCACGUTT-3' \\
& 5'-ACGUGACACGUUCGGAGAATT-3' \\
miR-138-5p inhibitor & 5'-CGGCCUGAUUCACAACACCAGCU-3' \\
NC inhibitor & 5'-CAGUACUUUUGUGUAGUACAA-3' \\
\hline
\end{tabular}

Table 2 The sequences of primers used for PCR

\begin{tabular}{ll}
\hline Name & Sequence \\
\hline HOTAIR F & 5'-TAGGCAAATGTCAGAGGGTT-3' \\
HOTAIR R & 5'-CTTAAATTGGGCTGGGTCT-3' \\
$\beta$-actin F & 5'-ACCCTGAAGTACCCCATCGA-3' \\
$\beta$-actin R & 5'-CAAACATGATCTGGGTCATCT-3' \\
hsa-miR-138-5p F & 5'-GCCGAGCTGGTGTTGTGAAT-3' \\
hsa-miR-138-5p R & 5'-GTGCAGGGTCCGAGGTATTC-3' \\
U6 F & 5'-GCTTCGGCAGCACATATACT-3' \\
U6 R & 5'-GTGCAGGGTCCGAGGTATTC-3' \\
\hline
\end{tabular}

inhibitor, NC inhibitor (a non-targeting miRNA inhibitor), si-HOTAIRs, or NC siRNA (a non-targeting siRNA) using Lipofectamine 2000 (Invitrogen, Carlsbad, CA, USA) according to the manufacturer's protocols. The sequences were listed in Table 1 . After $48 \mathrm{~h}$ post-transfection, drug-resistant cells were harvested for further analyses.

\section{RNA extraction and real-time PCR analysis}

Total RNAs were extracted from cells with the TRIpure reagent (BioTeke, Beijing, China) according to the manufacturer's instructions. Then cDNAs were synthesized using Super M-MLV reverse transcriptase (BioTeke). Real-time PCR reactions were performed in a mixture of $20 \mu \mathrm{l}$ consisting $1 \mu \mathrm{l}$ cDNA, $0.5 \mu \mathrm{l}$ of $10 \mu \mathrm{M}$ forward/reverse primer, $0.3 \mu \mathrm{l}$ of SYBR Green, and $10 \mu \mathrm{l}$ of $2 \times$ Power Taq PCR using an Exicycler 96 Real-Time Quantitative Thermal Block (Bioneer, Korea). Data were collected and analyzed by the $2^{-\Delta \Delta C T}$ method. The sequences of primers used for PCR were listed in Table 2. We used beta-actin as an internal reference for HOTAIR 
detection based on the geNorm analysis [23]. U6 was used as an internal control for miR-138-5p detection.

\section{MTT assay}

Cells $\left(5 \times 10^{3}\right.$ cells per well) were seeded in 96 -well plates. DDP with a range of concentrations $(0 \mu \mathrm{M}, 5 \mu \mathrm{M}, 10 \mu \mathrm{M}$, $20 \mu \mathrm{M}, 40 \mu \mathrm{M}, 80 \mu \mathrm{M})$ was added into each group. The plates were then incubated at $37^{\circ} \mathrm{C}$ for an additional $48 \mathrm{~h}$. Thereafter, the culture solutions were replaced by new medium with MTT $(0.5 \mathrm{mg} / \mathrm{ml})$ and the plates were then incubated for $4.5 \mathrm{~h}$ at $37^{\circ} \mathrm{C}$ with $5 \% \mathrm{CO}_{2}$. After that, the culture solutions were carefully removed and $150 \mu$ lof DMSO was added into each well to completely dissolve the generated formazan crystals. The absorbance was measured at a wavelength of $570 \mathrm{~nm}$ using a microplate absorbance reader (BioTek, Winooski, VT, USA).

\section{Western blot}

Cells were lysed with RIPA lysis buffer (Beyotime Institute of Biotechnology, Shanghai, China) to obtain total proteins. Protein concentration was determined using the BCA Protein Assay Kit (Beyotime Institute of Biotechnology). Protein samples $(20-40 \mu \mathrm{g})$ were subjected to sodium dodecyl sulfate-polyacrylamide gel electrophoresis (SDS-PAGE), transferred to polyvinylidene fluoride (PVDF) membranes (Millipore, Massachusetts, USA), and blocked with 5\% skimmed milk. After that, the membrane was treated with primary antibodies against Enhancer of zeste 2 polycomb repressive complex 2 subunit (EZH2; 1:1000, CST, Danvers, MA, USA), sirtuin 1 (SIRT1; 1:1000, CST), Bcl2 (1:500, Beyotime Institute of Biotechnology), Bax (1:500, Beyotime Institute of Biotechnology), cleaved caspase 3 (CI-caspase 3; 1:000, Beyotime Institute of Biotechnology), cleaved PARP (CI-PARP; 1:000, Beyotime Institute of Biotechnology), and $\beta$-actin (1:1000, Santa Cruz Biotechnology, Santa Cruz, CA, USA) overnight at $4{ }^{\circ} \mathrm{C}$, followed by incubation of horseradish peroxidase-conjugated goat anti-rabbit or anti-mouse secondary antibodies (1:5000, Beyotime Institute of Biotechnology) for $45 \mathrm{~min}$ at $37^{\circ} \mathrm{C}$. Then, enhanced chemiluminescence (ECL) chromogenic substrates were used for protein visualization with a gel imaging system (Beijing Liuyi Instrument Factory, Beijing, China), and the protein bands were quantified by optical densitometry (Gel-Pro-Analyzer software).

\section{Apoptosis assay}

Apoptosis was measured using an Annexin V/FITC and propidium iodide (PI) apoptosis detection kit (Keygen, Nanjing, China). Briefly, collected cells were gently resuspended in $500 \mu \mathrm{l}$ of binding buffer. Then, cells were incubated with $5 \mu \mathrm{l}$ Annexin V-FITC and $5 \mu \mathrm{l}$ PI for $10 \mathrm{~min}$ away from light. The cells were analyzed by a flow cytometer (Aceabio, San Diego, CA, USA).

\section{Luciferase assay}

Wild type fragments of HOTAIR, EZH2 3 'UTR and SIRT1 3 'UTR containing putative binding sites for miR138-5p or mutant-type fragments (putative binding sites for miR-138-5p were mutated) were cloned into the pmirGLO vector between SalI and NheI restriction enzyme sites. The constructed plasmids and miR-138-5p mimic were co-transfected into 293T cells for $48 \mathrm{~h}$ using Lipofectamine 2000 (Invitrogen). Firefly and Renilla luciferase activities were determined using the dual luciferase assay system (Promega, Madison, WI, USA), and firefly luciferase activity was normalized to Renilla luciferase activity, according to the manufacturer's directions.

\section{Statistical analysis}

Data are presented as means \pm standard errors. Student's t-test and one-way analysis of variance were used to determine the significance between groups. Statistical analysis was carried out using Graphpad Prism 7. Differences with $\mathrm{p}$-values of $<0.05$ were considered statistically significant.

\section{Results \\ Expression levels of HOTAIR and miR-138-5p in DDP-resistant ovarian cancer cells}

In order to investigate the effect of HOTAIR/miR-138-5p axis on DDP-resistance in ovarian cancer, two cisplatin chemo-resistant ovarian cell lines (SKOV3/DDP and A2780/DDP) were constructed by treatment of SKOV3 and A2780 cells (the two mostly commonly used cellular models of ovarian cancer) with DDP $(8 \mu \mathrm{M})$ for 12 weeks. The half-maximal inhibitory concentration $\left(\mathrm{IC}_{50}\right)$ values of both DDP-sensitive (SKOV3 and A2780) and DDPresistant (SKOV3/DDP and A2780/DDP) cells were determined by MTT assay. As shown in Fig. 1a, SKOV3/ DDP and A2780/DDP cells performed significantly enhanced DDP-resistance (" $\mathrm{p}<0.05)$. The calculated IC50 values of SKOV3 cell line increased significantly from $9.18 \pm 0.84 \mu \mathrm{M}$ to $34.86 \pm 4.53 \mu \mathrm{M}\left({ }^{*} \mathrm{p}<0.001\right)$, and the $\mathrm{IC}_{50}$ values of A2780 cell line increased remarkably from $6.55 \pm 0.91 \mu \mathrm{M}$ to $58.53 \pm 6.89 \mu \mathrm{M}\left({ }^{*} \mathrm{p}<0.001\right)$. Furthermore, the expression of HOTAIR and miR-138-5p in DDP-resistant cells was detected by real-time PCR. As shown in Fig. 1b, the expression levels of HOTAIR were increased in both SKOV3/DDP and A2780/DDP cell lines $(* \mathrm{p}<0.05)$. Inversely, the expression levels of miR-138-5p were decreased in DDP-resistant cell lines ("p $<0.05)$ (Fig. 1c). The results suggested that HOTAIR and miR-138-5p might be implicated in the development of DDP resistance of ovarian cancer cells. Moreover, the 
Chang et al. Biol Res $\quad$ (2020) 53:18

Page 4 of 10

a

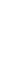

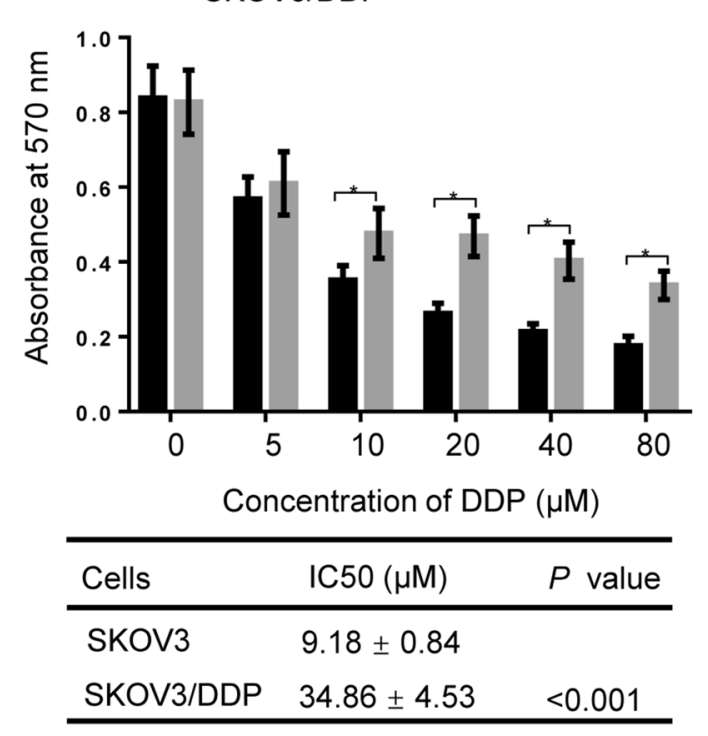

b

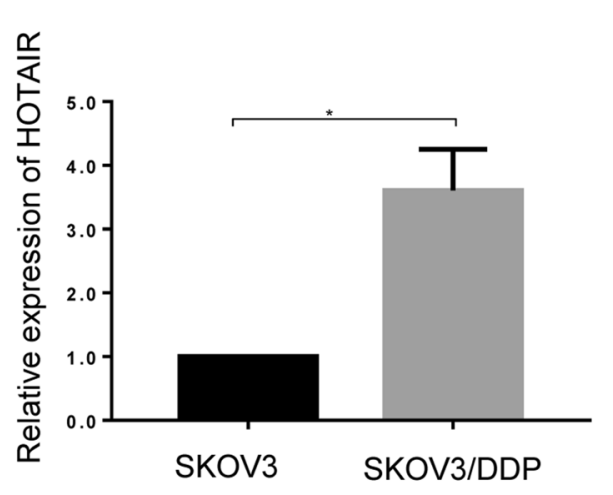

c

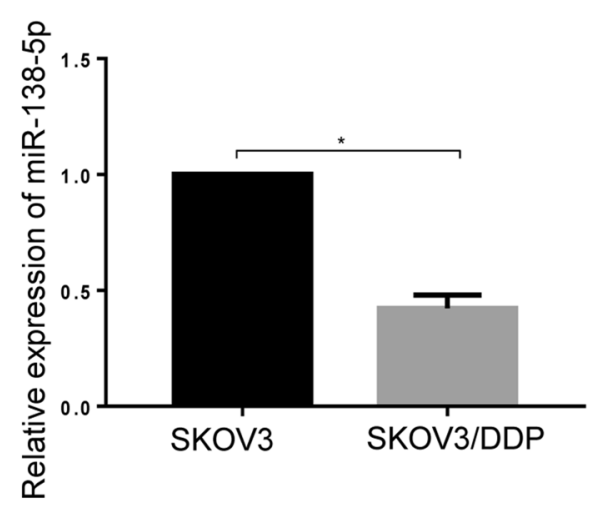

SKOV3/DDP
SKOV3
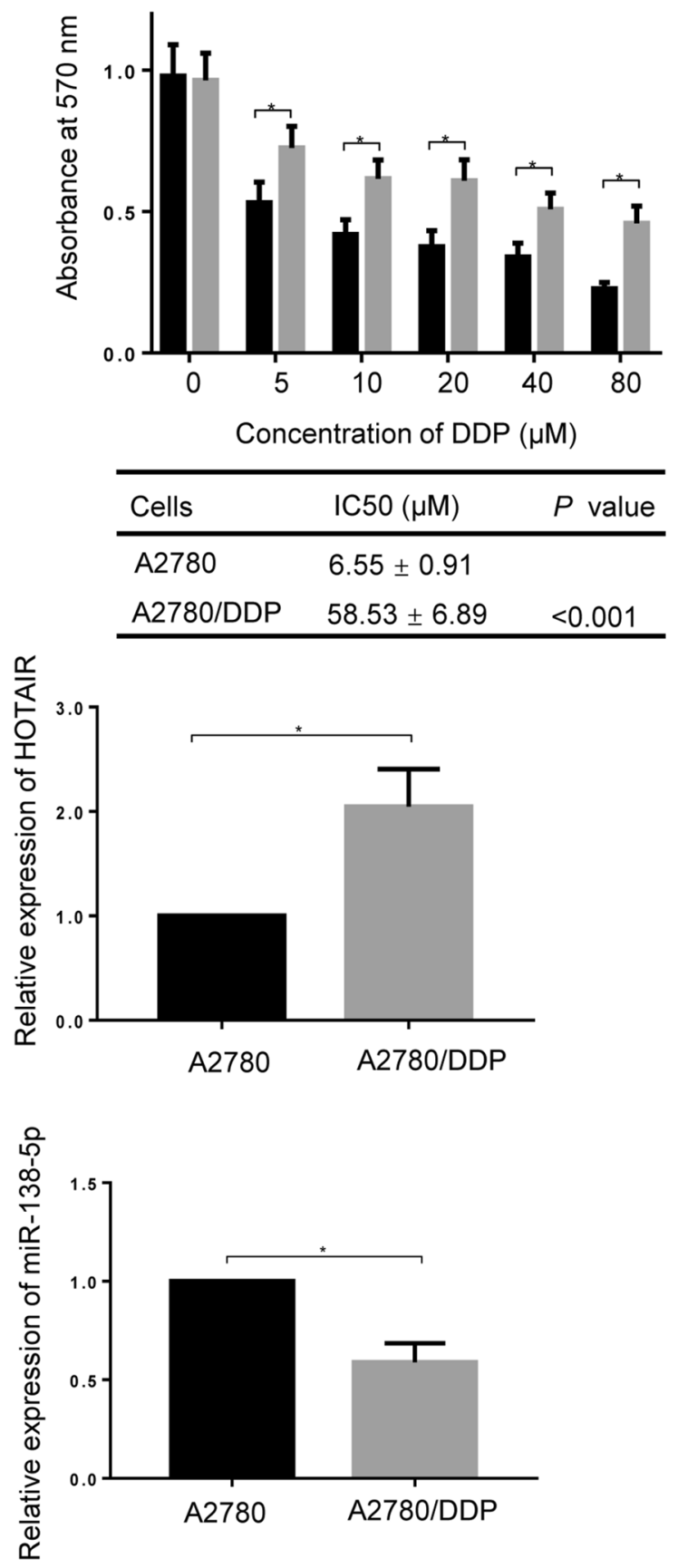

Fig. 1 Expression levels of HOTAIR and miR-138-5p in DDP-resistant ovarian cancer cells. a The effect of different concentrations of DDP on cell viability in DDP sensitive (SKOV3 and A2780) and DDP resistant (SKOV3/DDP and A2780/DDP) cells was determined by MTT assay, and the IC50 value was obtained. Real-time PCR was employed to evaluate the expression of $\mathbf{b}$ HOTAIR and $\mathbf{c}$ mir-138-5p in DDP sensitive (SKOV3 and A2780) and DDP resistant (SKOV3/DDP and A2780/DDP) cells, beta-actin and U6 were used as an internal control for HOTAIR and miR-138-5p detection, respectively. Data were presented as mean \pm SD. ${ }^{*} p<0.05$ 
a

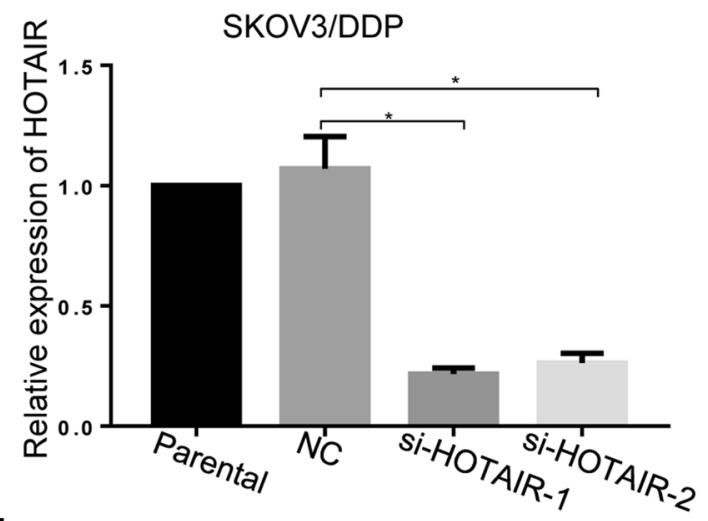

b

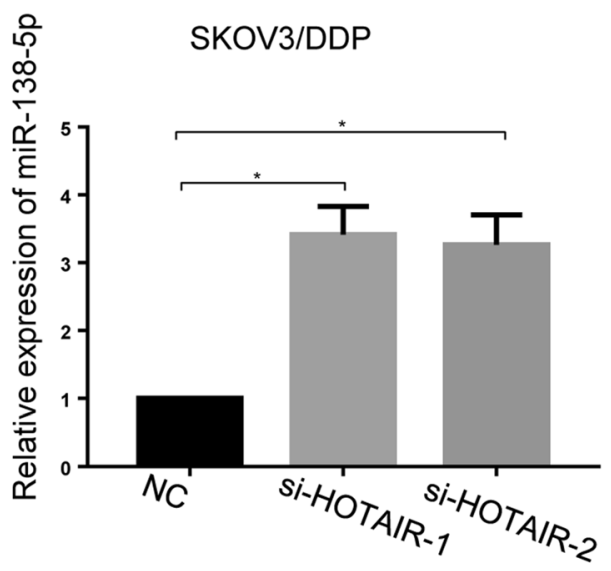

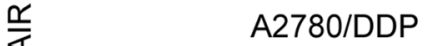
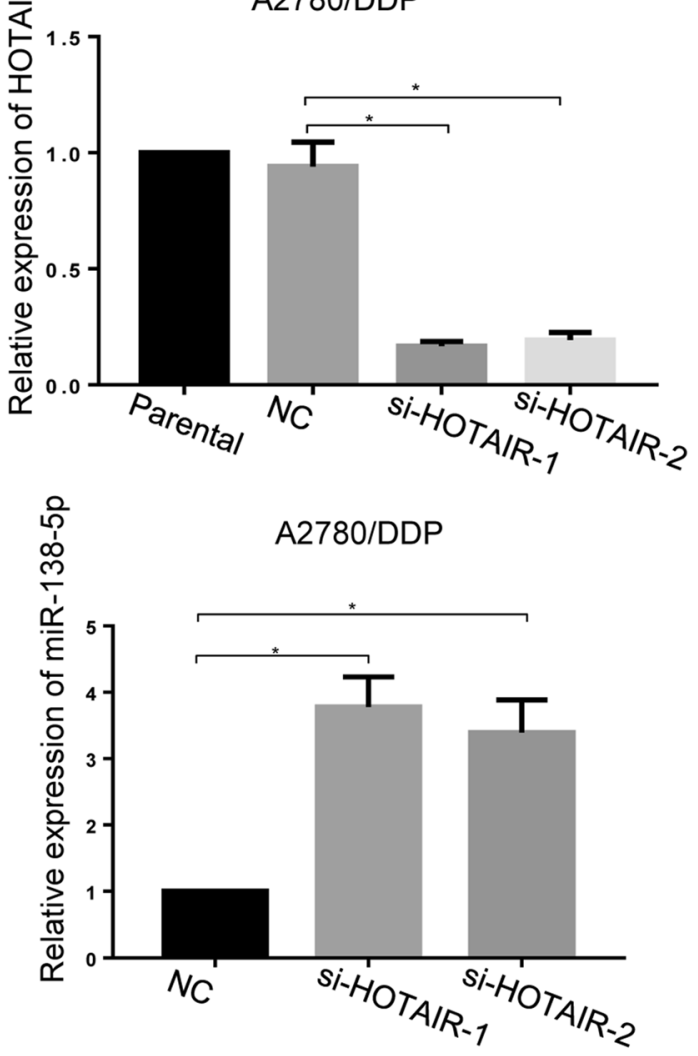

C

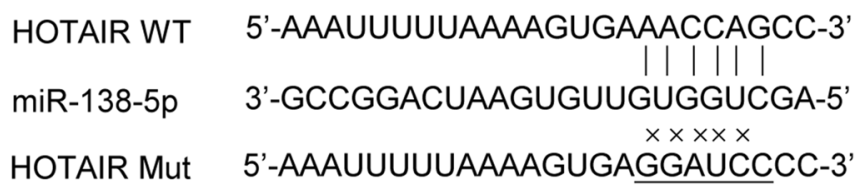

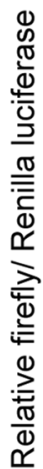

Fig. 2 Knockdown of HOTAIR increased miR-138-5p expression in DDP-resistant ovarian cancer cells. a Downregulation of HOTAIR by two different small interfering RNAs. b The expression of miR-138-5p in DDP-resistant ovarian cancer cells after silencing of HOTAIR was assessed by real-time PCR, beta-actin and U6 were used as an internal control for HOTAIR and miR-138-5p detection, respectively. c Dual-luciferase assay was used to verify the direct binding of miR-138-5p and HOTAIR. Data were presented as mean \pm SD. ${ }^{*} p<0.05$ 
a

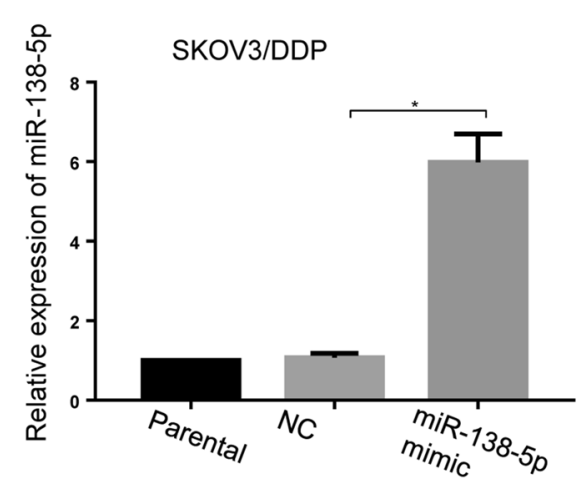

b

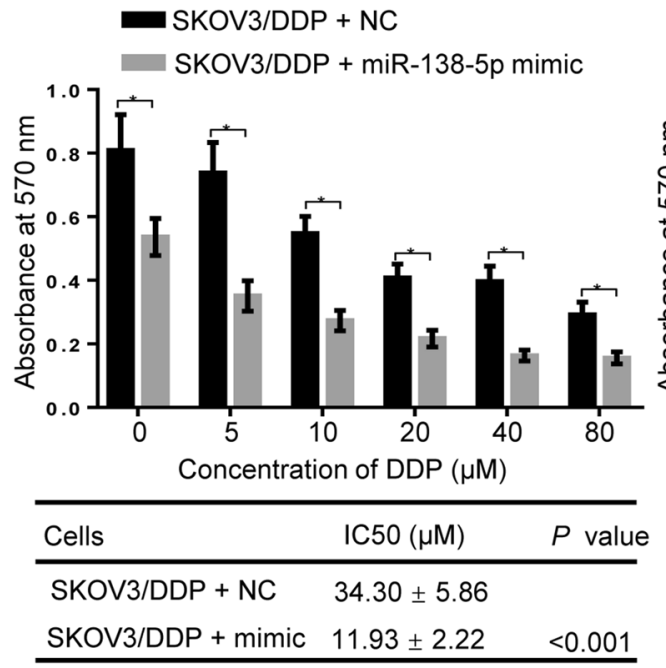

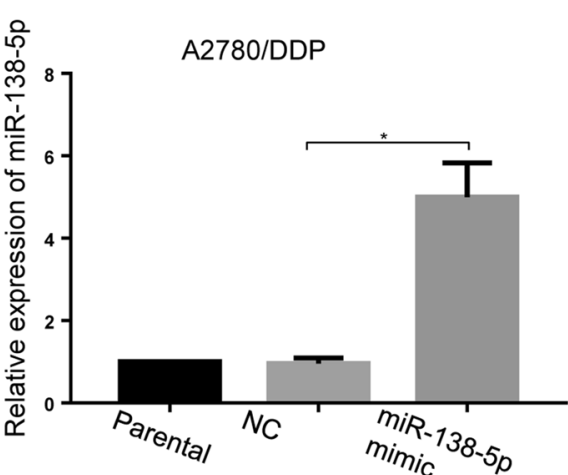

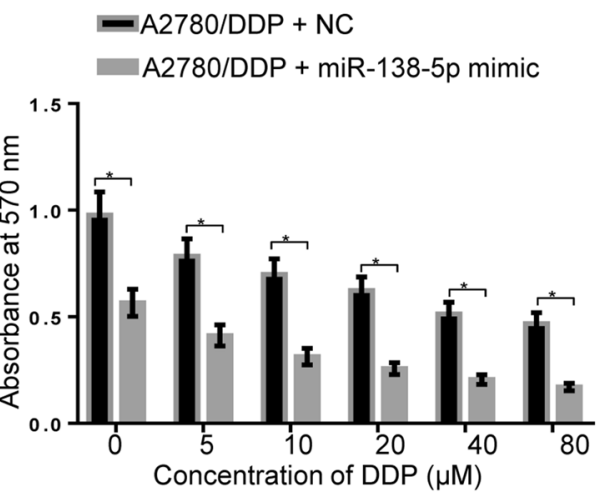

\begin{tabular}{lll}
\hline Cells & IC50 $(\mu \mathrm{M})$ & $P$ value \\
\hline A2780/DDP + NC & $58.17 \pm 8.42$ & \\
A2780/DDP + mimic & $18.09 \pm 2.07$ & $<0.001$ \\
\hline
\end{tabular}

Fig. 3 Upregulation of miR-138-5p enhanced chemosensitivity of DDP-resistant ovarian cancer cells to DDP. a Verification of overexpression of miR-138-5p by real-time PCR assay, U6 were used as an internal control. b The effect of different concentrations of DDP on cell viability in SKOV3/ DDP and A2780/DDP cells with or without forced overexpression of miR-138-5p was assessed by MTT assay, and the IC50 value was obtained. Data were presented as mean \pm SD. ${ }^{*} p<0.05$

negative correlation between the expression of HOTAIR and mir-138-5p might indicate potential binding in DDPresistant ovarian cancer cells

\section{HOTAIR targets and modulates the expression of miR-138-5p}

The relationship between HOTAIR and miR-138-5p expression was further explored. Two ovarian cancer DDP-resistant cell lines (SKOV3/DDP and A2780/DDP) were transfected with designed HOTAIR small interfering RNA fragments (si-HOTAIR1 and si-HOTAIR2) to silence the expression of HOTAIR. The effectiveness was verified by real-time PCR experiments (Fig. 2a). Compared with negative controls, knockdown of HOTAIR dramatically increased the expression of miR-138-5p (Fig. 2b) ("p<0.05). Bioinformatics prediction for potential binding sites between HOTAIR and miR-138-5p was shown in Fig. 2c. Moreover, the relative luciferase activities in cells co-transfected with HOTAIR WT and miR-138-5p mimic (which is a double-stranded RNA consisting of the guide strand that is designed to mimic the function of the endogenous miRNA and the passenger strand that is partially complementary to the guide strand) was significantly downregulated (* $\mathrm{p}<0.05)$, indicating that HOTAIR can bind to miR-138-5p (Fig. 2c).

\section{MiR-138-5p positively regulates DDP sensitivity of ovarian cancer cells}

SKOV3/DDP and A2780/DDP cells were transfected with miR-138-5p mimic or negative control, and transfection efficiency was detected after $48 \mathrm{~h}$ by real-time PCR. As shown in Fig. 3a, the relative expression of miR-138-5p in both SKOV3/DDP and A2780/DDP cells was significantly increased following miR-138-5p mimic treatment (* $\mathrm{p}<0.05)$. After that, the influence of DDP on cell viability in SKOV3/DDP and A2780/DDP cells 

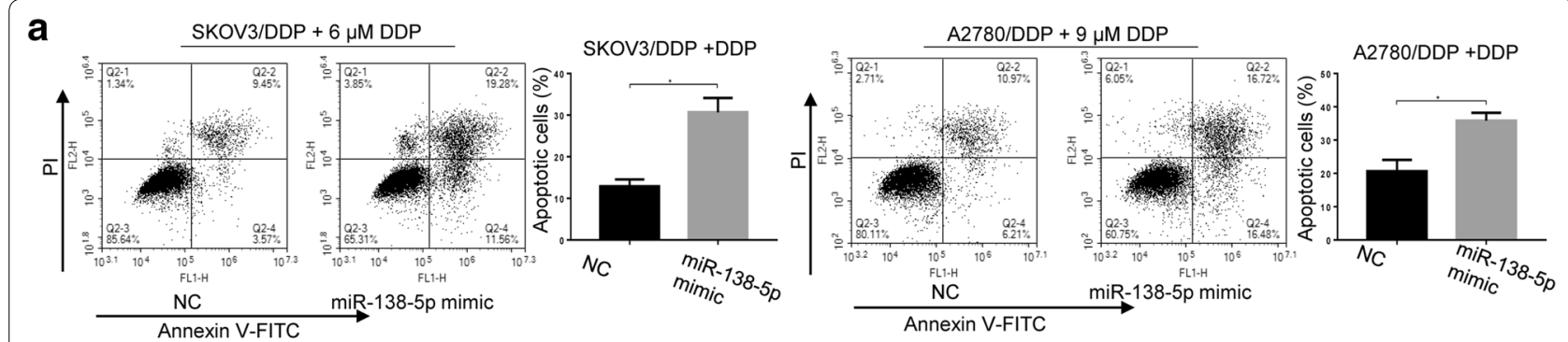

b

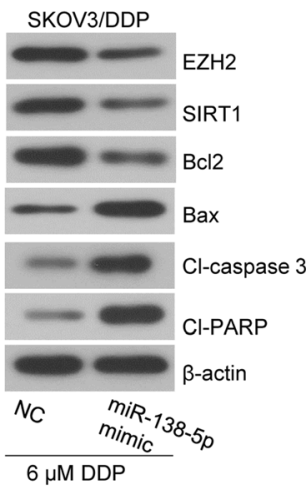

C

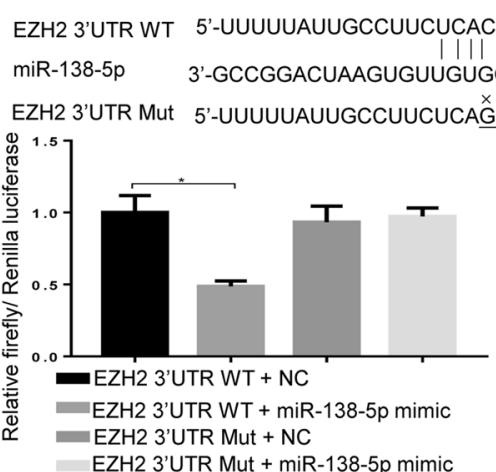

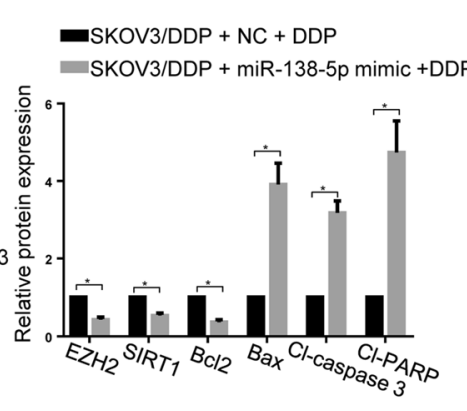
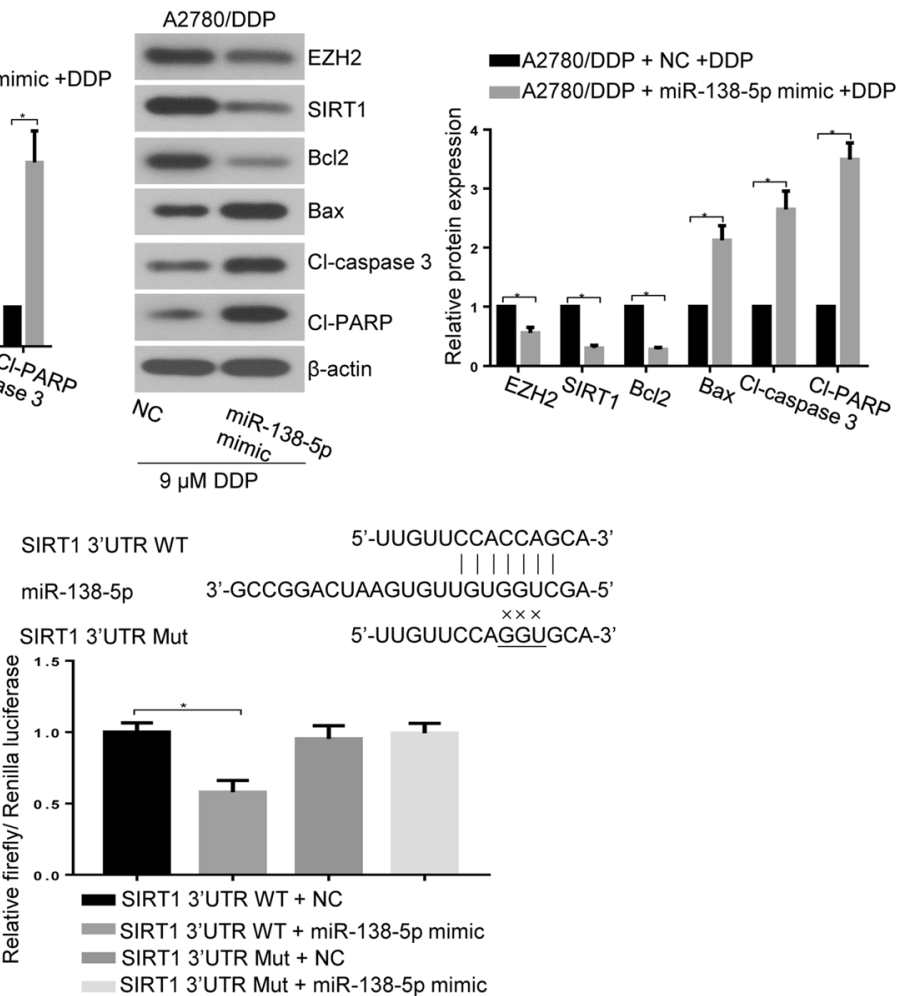

Fig. 4 Upregulation of miR-138-5p promoted DDP-mediated apoptosis in DDP-resistant ovarian cancer cells. a Cell apoptosis in DDP-treated SKOV3/DDP and A2780/DDP cells with miR-138-5p overexpression was determined by annexin V-FITC and PI staining through flow cytometry. $\mathbf{b}$ Expression levels of EZH2, SIRT1, Bcl2, Bax, Cl-caspase 3 and Cl-PARP were evaluated by western blot analysis. c Dual-luciferase assay was used to verify the direct binding of miR-138-5p with EZH2 and SIRT1. Data were presented as mean \pm SD. * $p<0.05$

with or without forced overexpression of miR-138-5p was measured by MTT assay. As shown in Fig. 3b, transfection with the miR-138-5p mimic remarkably inhibited cell proliferation compared to transfection with the negative control (* $\mathrm{p}<0.05)$. The calculated $\mathrm{IC}_{50}$ values of transfected SKOV3/DDP cell line decreased significantly from $34.30 \pm 5.86 \mu \mathrm{M}$ to $11.93 \pm 2.22 \mu \mathrm{M}($ " $\mathrm{p}<0.001)$, and the $\mathrm{IC}_{50}$ values of $\mathrm{A} 2780 / \mathrm{DDP}$ cell line decreased significantly from $58.17 \pm 8.42 \mu \mathrm{M}$ to $18.09 \pm 2.07 \mu \mathrm{M}$ ("p $<0.001)$.

To further explore whether miR-138-5p positively regulated cell apoptosis of DDP-resistant ovarian cancer cells, SKOV3/DDP and A2780/DDP cells were transfected with miR-138-5p mimic, followed by DDP treatment (6 $\mu \mathrm{M}$ for SKOV3/DDP and $9 \mu \mathrm{M}$ for A2780/DDP). As shown in Fig. 4a, flow cytometry analysis revealed that the increase in apoptotic rate was evidently proved after overexpressing miR-138-5p in SKOV3/DDP and A2780/ DDP cells $(* \mathrm{p}<0.05)$. Moreover, western blot analysis showed that the protein levels of EZH2, SIRT1 and Bcl2 were markedly decreased in DDP-treated drug-resistant cells following miR-138-5p upregulation, while protein expression levels of Bax, $\mathrm{Cl}$-caspase 3 and Cl-PARP were increased (Fig. 4b) (" $\mathrm{p}<0.05)$. The dual-luciferase assay showed that miR-138-5p could directly bind to the $3^{\prime}$ UTR of EZH2 and SIRT1 (Fig. 4c) ("p<0.05). Taken 


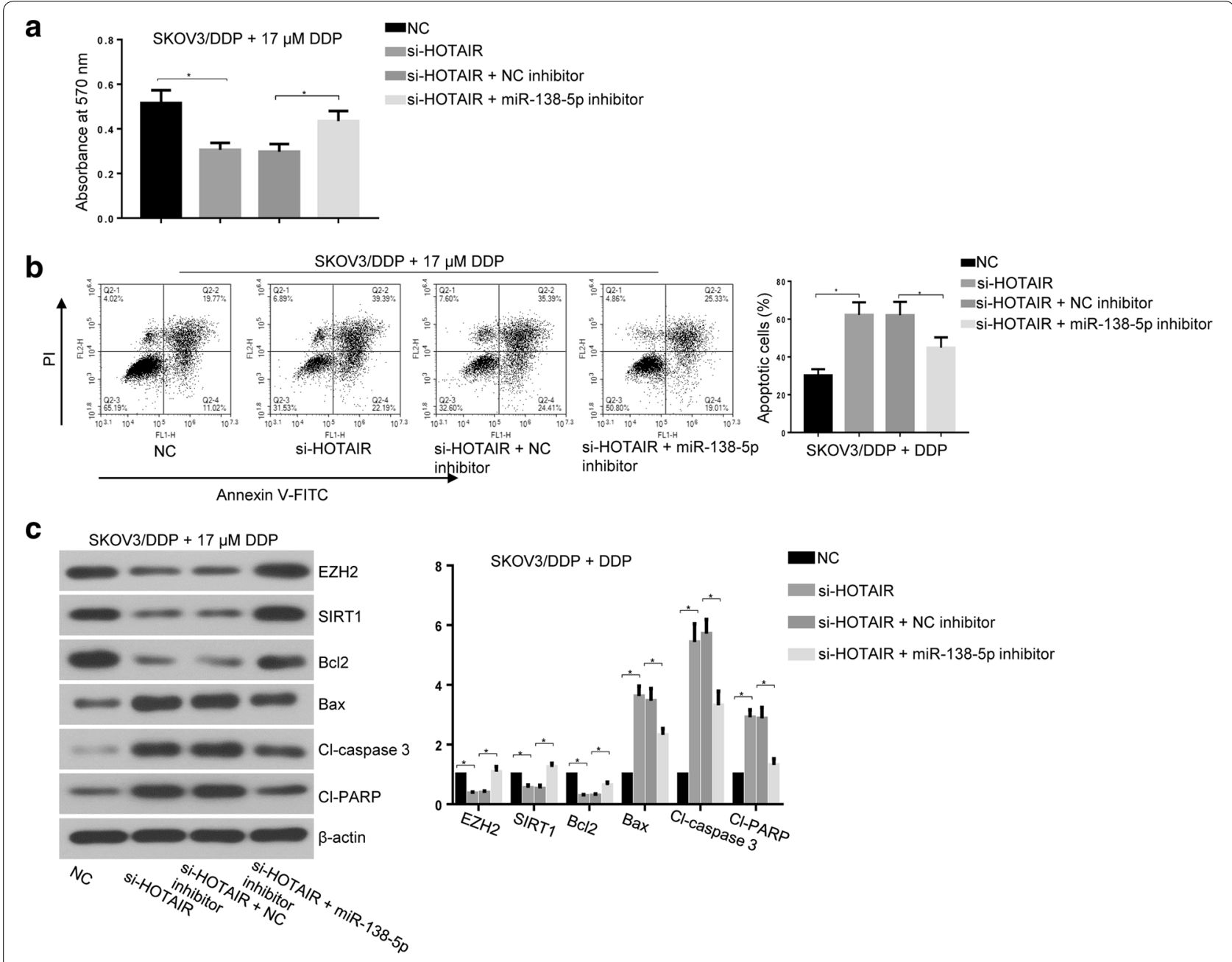

Fig. 5 Downregulation of miR-138-5p reversed silencing of HOTAIR mediated pro-apoptosis activity in DDP-treated SKOV3/DDP cells. a Cell viability in DDP-treated SKOV3/DDP cells with HOTAIR silencing or/and miR-138-5p silencing was determined by MTT assay. $\mathbf{b}$ Cell apoptosis was assessed by annexin V-FITC and PI staining with flow cytometry. c Expression levels of EZH2, SIRT1, Bcl2, Bax, Cl-caspase 3 and Cl-PARP were assessed by western blot assay. Data were presented as mean \pm SD. * $p<0.05$

together, the results indicated that the forced expression of miR-138-5p increased the cisplatin chemosensitivity.

\section{Downregulation of HOTAIR contributes to DDP-sensitivity} of ovarian cancer cells by upregulation of miR-138-5p

Knockdown of HOTAIR inhibited cell viability and promoted cell apoptosis in DDP-treated SKOV3/DDP cells (Fig. 5a, b), which was accompanied by the decrease of EZH2, SIRT1 and Bcl2 and by the increase of Bax, Clcaspase 3 and Cl-PARP (Fig. 5c). Furthermore, silencing of miR-138-5p reversed the effect of HOTAIR knockdown on chemosensitivity of SKOV3/DDP cells to DDP (Fig. 5a-c). Overall, these data suggested that HOTAIR modulates cisplatin chemosensitivity of ovarian cancer cells by miR-138-5p.

\section{Discussion}

Ovarian cancer is the leading cause of death in gynecologic cancers, and DDP-based chemotherapy is one of the first-line therapeutic approaches for ovarian cancer $[24,25]$. However, chemotherapy resistance strikingly decreased therapeutic efficiency and contributed to a high relapse rate in ovarian cancer [26]. Therefore, it is of importance to illuminate the molecular mechanism underlying the DDP-resistance for improvement of the prognosis.

LncRNAs can function as a competing endogenous RNA (ceRNA) by sponging miRNAs to regulate gene expression at the post-transcriptional level. HOTAIR regulates HER2 expression by sponging miR-331-3p in gastric cancer [27]. HOTAIR regulates CCND1 and CCND2 expression by sponging miR-206 in ovarian cancer [28]. 
Previous studies have been reported that HOTAIR was negatively correlated with DDP-resistance in gastric cancer [29-31], human lung adenocarcinoma [32], and small cell lung cancer [33]. For instance, HOTAIR was considered to play an important role in gastric cancer by PI3K/ Akt and Wnt/ $\beta$-catenin signaling pathways by up-regulating miR-34a. Knockdown of HOTAIR increased the inhibitory effect of DDP on tumor growth in vivo [29]. However, the molecular mechanism of HOTAIR in DDP resistance of ovarian cancer remains undefined.

Clinical studies demonstrated that the expression levels of miR-138-5p were significantly upregulated in patients with ovarian cancer [34]. Moreover, Chen et al. [18] indicated that miR-138 could directly target LIM kinase 1 (Limk1) and inhibited metastasis of ovarian cancer by Limk1/cofilin/p-cofilin signaling pathway. Yeh et al. [35] pointed out that miR-138 regulated ovarian cancer cell invasion and metastasis by targeting SOX4 and HIF-1a oncogenic transcriptional factors. Unfortunately, the roles of miR-138-5p in DDP-resistance in ovarian cancer cells remain unclear. In this study, we successfully obtained DDP-resistant ovarian cancer cells (SKOV3/DDP and A2780/DDP). Increased HOTAIR and decreased miR-138-5p expression were found in SKOV3/DDP and A2780/DDP cells. Knockdown of HOTAIR could increase the expression of miR-138-5p, which could enhance the chemosensitivity of DDP-resistance cells to DDP. Moreover, we demonstrated that miR-138-5p could directly regulate the expression of EZH2 and SIRT1, which have been proven to be involved in regulation of cisplatin resistance of ovarian cancer [36, 37]. Interestingly, our data showed that downregulation of miR-138-5p only partially reversed the HOTAIR silencing-mediated pro-apoptosis activity, suggesting that other miRNAs or molecules may also contribute to the action of HOTAIR on chemosensitivity of ovarian cancer cells to DDP. Many miRNAs (such as miR-34a and miR-454) reported to be involved in regulating the chemosensitivity of ovarian cancer were regulated by HOTAIR [38-41], indicating that HOTAIR may also mediate chemoresistance of ovarian cancer cells by other miRNAs and their target genes. Moreover, the efficacy of HOTAIR/miR-138-5p axis on the treatment in clinical studies needs further explorations.

\section{Conclusion}

In the present study, we demonstrated that HOTAIR/ miR-138-5p axis could regulate the DDP-resistance of ovarian cancer by potential targets EZH2 and SIRT1, which could shed light on new therapeutic targets for ovarian cancer treatment.

\section{Abbreviations}

DDP: Cisplatin; LncRNA: Long non-coding RNA; HOTAIR: HOX transcript antisense RNA; EZH2: Enhancer of zeste 2 polycomb repressive complex 2 subunit; SIRT1: Sirtuin 1; miR-138-5p: MicroRNA-138-5p.

\section{Acknowledgements}

Not applicable.

\section{Authors' contributions}

LLL and ZY conceived and designed the experiments; ZY, FX, CSX, WYD performed experiments; $Z Y$ and $A H$ analyzed the data; LLL and ZY wrote the manuscript. All authors read and approved the final manuscript.

\section{Funding}

This study was supported by a Grant from the Natural Science Foundation of Liaoning Province (No. 20170540339).

\section{Availability of data and materials}

The datasets used and/or analyzed during the current study are available from the corresponding author on reasonable request.

\section{Ethics approval and consent to participate}

Not applicable.

\section{Consent for publication}

Not applicable.

\section{Competing interests}

The authors declare that they have no competing interests.

\section{Author details \\ ${ }^{1}$ Department of Obstetrics and Gynecology, The First Affiliated Hospital of Jinzhou Medical University, No. 2, Section 5, Renmin Street, Jinzhou 121001, Liaoning, People's Republic of China. ${ }^{2}$ Liaoning Key Laboratory of Follicular Development and Reproductive Health, Jinzhou 121001, Liaoning, People's Republic of China. ${ }^{3}$ Department of Obstetrics and Gynecology, The Third Affiliated Hospital of Jinzhou Medical University, Jinzhou 121002, Liaoning, People's Republic of China. ${ }^{4}$ Department of Pathology, The Third Affiliated Hospital of Jinzhou Medical University, Jinzhou 121002, Liaoning, People's Republic of China. ${ }^{5}$ Department of Oncology, The Third Affiliated Hospital of Jinzhou Medical University, Jinzhou 121002, Liaoning, People's Republic of China.}

Received: 28 July 2019 Accepted: 17 April 2020

Published online: 29 April 2020

\section{References}

1. Siegel RL, Miller KD, Jemal A. Cancer statistics, 2018. CA Cancer J Clin. 2018;68(1):7-30.

2. Agarwal R, Kaye SB. Ovarian cancer: strategies for overcoming resistance to chemotherapy. Nat Rev Cancer. 2003;3(7):502-16.

3. Clamp AR, James EC, McNeish IA, Dean A, Kim JW, O’Donnell DM, et al. Weekly dose-dense chemotherapy in first-line epithelial ovarian, fallopian tube, or primary peritoneal carcinoma treatment (ICON8): primary progression free survival analysis results from a GCIG phase 3 randomised controlled trial. Lancet. 2019;394(10214):2084-95.

4. Dall'Acqua A, Sonego M, Pellizzari I, Pellarin I, Canzonieri V, D'Andrea S, et al. CDK6 protects epithelial ovarian cancer from platinum-induced death via FOXO3 regulation. EMBO Mol Med. 2017;9(10):1415-33.

5. Louro R, Smirnova AS, Verjovski-Almeida S. Long intronic noncoding RNA transcription: expression noise or expression choice? Genomics. 2009;93(4):291-8.

6. Loewer S, Cabili MN, Guttman M, Loh Y-H, Thomas K, Park IH, et al. Large intergenic non-coding RNA-RoR modulates reprogramming of human induced pluripotent stem cells. Nat Genet. 2010;42(12):1113-7.

7. Wilusz JE, Sunwoo H, Spector DL. Long noncoding RNAs: functional surprises from the RNA world. Gene Dev. 2009;23(13):1494-504.

8. Yuan S-X, Yang F, Yang Y, Tao Q-F, Zhang J, Huang G, et al. Long noncoding RNA associated with microvascular invasion in hepatocellular carcinoma 
promotes angiogenesis and serves as a predictor for hepatocellular carcinoma patients' poor recurrence-free survival after hepatectomy. Hepatology. 2012;56(6):2231-41.

9. Du Y, Kong G, You X, Zhang S, Zhang T, Gao Y, et al. Elevation of highly up-regulated in liver cancer (HULC) by hepatitis B virus $X$ protein promotes hepatoma cell proliferation via down-regulating p18. J Biol Chem. 2012;287(31):26302-11.

10. Lu X, Fang Y, Wang Z, Xie J, Zhan Q, Deng X, et al. Downregulation of gas 5 increases pancreatic cancer cell proliferation by regulating CDK6. Cell Tissue Res. 2013;354(3):891-6.

11. Rinn JL, Kertesz M, Wang JK, Squazzo SL, Xu X, Brugmann SA, et al. Functional demarcation of active and silent chromatin domains in human HOX loci by noncoding RNAs. Cell. 2007;129(7):1311-23.

12. Gupta RA, Shah N, Wang KC, Kim J, Horlings HM, Wong DJ, et al. Long non-coding RNA HOTAIR reprograms chromatin state to promote cancer metastasis. Nature. 2010;464(7291):1071-6.

13. Zhao W, Song M, Zhang J, Kuerban M, Wang H. Combined identification of long non-coding RNA CCAT1 and HOTAIR in serum as an effective screening for colorectal carcinoma. Int J Clin Exp Pathol. 2015:8(11):14131-40.

14. Yang S-Z, Xu F, Zhou T, Zhao X, McDonald JM, Chen Y. The long non-coding RNA HOTAIR enhances pancreatic cancer resistance to TNF-related apoptosis-inducing ligand. J Biol Chem. 2017;292(25):10390-7.

15. Li J, Yang S, Su N, Wang Y, Yu J, Qiu H, et al. Overexpression of long non-coding RNA HOTAIR leads to chemoresistance by activating the Wnt/beta-catenin pathway in human ovarian cancer. Tumor Biol. 2016;37(2):2057-65

16. Liu S, Lei H, Luo F, Li Y, Xie L. The effect of IncRNA HOTAIR on chemoresistance of ovarian cancer through regulation of HOXA7. Biol Chem. 2018;399(5):485-97.

17. Qu M, Zhu Y, Jin M. MicroRNA-138 inhibits SOX12 expression and the proliferation, invasion and migration of ovarian cancer cells. Expe Ther Med. 2018;16(3):1629-38.

18. Chen P, Zeng M, Zhao Y, Fang X. Upregulation of Limk1 caused by microRNA-138 loss aggravates the metastasis of ovarian cancer by activation of Limk1/cofilin signaling. Oncol Rep. 2014;32(5):2070-6.

19. Mihanfar A, Fattahi A, Nejabati HR. MicroRNA-mediated drug resistance in ovarian cancer. J Cell Physiol. 2019;234(4):3180-91.

20. Wen W, Lajuan Z, Yexiong T, Haoren L, Zhongtian Q. MiR-138 induces cell cycle arrest by targeting cyclin D3 in hepatocellular carcinoma. Carcinogenesis. 2012;33(5):1113-20.

21. Huijun Z, Hui Z, Mingchuan Z, Zhongwei L, Xiaoping Z, Xiong Q, et al. MiR-138 inhibits tumor growth through repression of EZH2 in non-small cell lung cancer. Cell Physiol Biochem. 2013;31(1):56-65.

22. Liu X, Lv X-B, Wang X-P, Sang Y, Xu S, Hu K, et al. MiR-138 suppressed nasopharyngeal carcinoma growth and tumorigenesis by targeting the CCND1 oncogene. Cell Cycle. 2012;11(13):2495-506.

23. Vandesompele J, De Preter K, Pattyn F, Poppe B, Van Roy N, De Paepe A, et al. Accurate normalization of real-time quantitative RT-PCR data by geometric averaging of multiple internal control genes. Genome Biol. 2002;3(7):RESEARCH0034.

24. Bell D, Berchuck A, Birrer M, Chien J, Cramer DW, Dao F, et al. Integrated genomic analyses of ovarian carcinoma. Nature. 2011;474(7353):609-15.

25. Armstrong DK, Bundy B, Wenzel L, Huang HQ, Baergen R, Lele S, et al. Intraperitoneal cisplatin and paclitaxel in ovarian cancer. N Engl J Med. 2006;354(1):34-43.

26. Samuel P, Mulcahy LA, Furlong F, McCarthy HO, Brooks SA, Fabbri M, et al. Cisplatin induces the release of extracellular vesicles from ovarian cancer cells that can induce invasiveness and drug resistance in bystander cells. Philos Trans R Soc Lond B Biol Sci. 2018;373(1737):20170065.
27. Liu XH, Sun M, Nie FQ, Ge YB, Zhang EB, Yin DD, et al. Lnc RNA HOTAIR functions as a competing endogenous RNA to regulate HER2 expression by sponging miR-331-3p in gastric cancer. Mol Cancer. 2014;13:92.

28. Chang L, Guo R, Yuan Z, Shi H, Zhang D. LncRNA HOTAIR regulates CCND1 and CCND2 expression by sponging miR-206 in ovarian cancer. Cell Physiol Biochem. 2018;49(4):1289-303.

29. Cheng C, Qin Y, Zhi Q, Wang J, Qin C. Knockdown of long non-coding RNA HOTAIR inhibits cisplatin resistance of gastric cancer cells through inhibiting the PI3K/Akt and Wnt/beta-catenin signaling pathways by upregulating miR-34a. Int J Biol Macromol. 2018;107:2620-9.

30. Su C, Li J, Cao Y, Xiao L, Shi C. Long noncoding RNA HOTAIR promotes cisplatin resistance in gastric cancer through promoting WIF-1 promoter methylation. Int J Clin Exp Med. 2017;10(2):2717-26.

31. Zhang E-b, Kong R, Yin D-d, You L-h, Sun M, Han L, et al. Long noncoding RNA ANRIL indicates a poor prognosis of gastric cancer and promotes tumor growth by epigenetically silencing of miR-99a/miR-449a. Oncotarget. 2014;5(8):2276-92.

32. Liu Z, Sun M, Lu K, Liu J, Zhang M, Wu W, et al. The long noncoding RNA HOTAIR contributes to cisplatin resistance of human lung adenocarcinoma cells via downregualtion of p21(WAF1/CIP1) expression. PLoS ONE. 2013;8(10):e77293.

33. Guo F, Cao Z, Guo H, Li S. The action mechanism of IncRNA-HOTAIR on the drug resistance of non-small cell lung cancer by regulating Wnt signaling pathway. Exp Ther Med. 2018;15(6):4885-9.

34. Ayaz L, Cayan F, Balci S, Gorur A, Akbayir S, Yaroglu HY, et al. Circulating microRNA expression profiles in ovarian cancer. J Obstet Gynaecol. 2014;34(7):620-4.

35. Yeh Y-M, Chuang C-M, Chao K-C, Wang L-H. MicroRNA-138 suppresses ovarian cancer cell invasion and metastasis by targeting SOX4 and HIF-1. Int J Cancer. 2013;133(4):867-78.

36. Li D, Wu QJ, Bi FF, Chen SL, Zhou YM, Zhao Y, et al. Effect of the BRCA1SIRT1-EGFR axis on cisplatin sensitivity in ovarian cancer. Am J Transl Res. 2016;8(3):1601-8.

37. Sun J, Cai X, Yung MM, Zhou W, Li J, Zhang Y, et al. miR-137 mediates the functional link between c-Myc and EZH2 that regulates cisplatin resistance in ovarian cancer. Oncogene. 2019;38(4):564-80.

38. Zuo Y, Zheng W, Liu J, Tang Q, Wang SS, Yang XS. MiR-34a-5p/PD-L1 axis regulates cisplatin chemoresistance of ovarian cancer cells. Neoplasma. 2020;67(1):93-101.

39. Cheng C, Qin Y, Zhi Q, Wang J, Qin C. Knockdown of long non-coding RNA HOTAIR inhibits cisplatin resistance of gastric cancer cells through inhibiting the PI3K/Akt and Wnt/beta-catenin signaling pathways by upregulating miR-34a. Int J Biol Macromol. 2018;107(Pt B):2620-9.

40. Bao X, Ren T, Huang Y, Sun K, Wang S, Liu K, et al. Knockdown of long non-coding RNA HOTAIR increases miR-454-3p by targeting Stat3 and Atg12 to inhibit chondrosarcoma growth. Cell Death Dis. 2017;8(2):e2605

41. Wang DY, Li N, Cui YL. Long non-coding RNA CCAT1 sponges miR-454 to promote chemoresistance of ovarian cancer cells to cisplatin by regulation of surviving. Cancer Res Treat. 2020. https://doi.org/10.4143/ crt.2019.498.

\section{Publisher's Note}

Springer Nature remains neutral with regard to jurisdictional claims in published maps and institutional affiliations. 